\title{
Axial-Compressive Behavior, Including Kink-Band Formation and Propagation, of Single $p$-Phenylene Terephthalamide (PPTA) Fibers
}

\author{
M. Grujicic, ${ }^{1}$ S. Ramaswami, ${ }^{1}$ J. S. Snipes, ${ }^{1}$ R. Yavari, ${ }^{1}$ C.-F. Yen, ${ }^{2}$ and B. A. Cheeseman ${ }^{2}$ \\ ${ }^{1}$ Department of Mechanical Engineering, Clemson University, Clemson, SC 29634-0921, USA \\ ${ }^{2}$ Army Research Laboratory, Weapons \& Materials Research Directorate, Proving Ground, Aberdeen, MD 21005-5069, USA \\ Correspondence should be addressed to M. Grujicic; gmica@clemson.edu
}

Received 21 May 2013; Revised 15 July 2013; Accepted 17 July 2013

Academic Editor: Gongnan Xie

Copyright (c) 2013 M. Grujicic et al. This is an open access article distributed under the Creative Commons Attribution License, which permits unrestricted use, distribution, and reproduction in any medium, provided the original work is properly cited.

\begin{abstract}
The mechanical response of $p$-phenylene terephthalamide (PPTA) single fibers when subjected to uniaxial compression is investigated computationally using coarse-grained molecular statics/dynamics methods. In order to construct the coarse-grained PPTA model (specifically, in order to define the nature of the coarse-grained particles/beads and to parameterize various components of the bead/bead force-field functions), the results of an all-atom molecular-level computational investigation are used. In addition, the microstructure/topology of the fiber core, consisting of a number of coaxial crystalline fibrils, is taken into account. Also, following our prior work, various PPTA crystallographic/topological defects are introduced into the model (at concentrations consistent with the prototypical PPTA synthesis/processing conditions). The analysis carried out clearly revealed (a) formation of the kink bands during axial compression; (b) the role of defects in promoting the formation of kink bands; (c) the stimulating effects of some defects on the fiber-fibrillation process; and (d) the detrimental effect of the prior compression, associated with fiber fibrillation, on the residual longitudinal-tensile strength of the PPTA fibers.
\end{abstract}

\section{Introduction}

Fibers of $p$-phenylene terephthalamide (PPTA), available commercially as Kevlar, Twaron, Technora, and so forth, are characterized by high specific axial stiffness and high specific tensile strength. These fibers are often referred to as "ballistic fibers" since they are commonly used in different ballisticand/or blast-protection systems. Such systems are required to provide a high level of penetration-resistance against largekinetic-energy projectiles, such as bullets, detonated-mineinduced soil ejecta, fragments/shrapnel from improvised explosive devices (IEDs), and shells or turbines. To satisfy this requirement, PPTA fibers are normally used as (a) filament constituents in twodimensional or threedimensional woven-fabric protective structures (e.g., "bulletproof vests"); (b) reinforcements in high-performance (typically, polymermatrix, "rigid-armor") composites; or (c) energy-absorbing reinforcements in resin-starved flexible armor-grade composites [1-4].
Given the complex behavior/functionality of the aforementioned protection systems, their design and development increasingly involve the use of computer-aided engineering (CAE) methods and tools. In turn, the latter employ material models to describe the behavior of fibers and structures under high-rate loading conditions. With the utility of the CAE methods and tools largely determined by the fidelity of these models, their development must account for the hierarchi$\mathrm{cal} /$ multiscale architecture of the fibers-based structures, as well as of the fibers themselves [5-8].

The present work deals with the compressive axial behavior of single PPTA fibers and the effect of this behavior on the global/macroscopic response of the ballistic- and blast-protection systems. Consequently, the main topics overviewed in the remainder of this section include (a) the basics of PPTA fibers; (b) PPTA microstructural hierarchy; and (c) overview of the recent materialmodels for PPTA fiberreinforced polymer-matrix composites. 


\subsection{The Basics of PPTA Fibers}

1.1.1. Classification. PPTA fibers can be categorized as polyamide-type (polymeric) fibers. Polyamides are generally divided into two main classes: (i) nonaromatic polyamides (e.g., nylon-6,6) and (ii) aromatic polyamides or aramids (e.g., Kevlar, Twaron, Technora, etc.). It is the aromatic polyamides which are the subject of the present work.

1.1.2. PPTA Molecules/Chains. The PPTA repeat units/mers appear in two distinct (a) trans ("on the opposite side"); and (b) cis ("on the same side") molecular-level conformations. These conformations are shown using a ball-andstick representation in Figures $1(\mathrm{a})$ and 1(b), respectively. To help with interpretation of the molecular structure, the chemical elements are labeled in Figure 1(a). Examination of Figures 1(a) and 1(b) reveals that the repeat unit consists of two phenylene rings/moieties $\left(\mathrm{C}_{6} \mathrm{H}_{4} \mathrm{RR}^{\prime}\right)$ connected by an amide linkage (-CO-NH-). Although the PPTA molecule can assume either the trans or cis stereo-isomeric conformations, the former conformation is almost exclusively observed. The reason for this predominance is that the trans conformation promotes the stretching/extension of PPTA molecules, lowering the system energy. In addition, extended molecules can readily align, forming sheets consisting of nearlyparallel regularlyspaced PPTA chains. Formation of these sheets is associated with further reductions in the system energy. Regular stacking of these sheets leads to the formation of low-energy crystalline PPTA fibrils (subfiber units, elongated and coaxial with the fibers). In sharp contrast, the cis conformation hampers the formation of these low-energy structures, which explains its rare occurrence.

1.1.3. PPTA Sheets. Due to a large difference in electronegativity between oxygen and hydrogen, the electron charge density within the PPTA unit cell is increased around the oxygen atoms and lowered around the hydrogen atoms, giving rise to the formation of (relatively strong) dipoles. The presence of dipoles along with (i) the lone electron pairs on the oxygen atoms; (ii) close proximity of the oxygen and hydrogen atoms of neighboring parallel PPTA molecules within the same sheet; and (iii) the operation of London-dispersion effects typically results in the formation of (strong) hydrogen bonds. Within these bonds, the $\mathrm{N}-\mathrm{H}$ species acts as a bond donor while $\mathrm{O}$ acts as a bond acceptor. The formation of the aforementioned sheet-like structures is believed to be the result of strong lateral hydrogen bonding between adjacent parallel PPTA molecules.

1.1.4. PPTA Fibrils. As mentioned previously, stacking of sheets results in the formation of crystalline fibrils, the axes of which are aligned with the fiber axis. However, orientation of the fibril crystal structure within a plane normal to the fiber axis varies among the fibrils. In other words, PPTA fibrils within a single fiber resemble columnar grains observed in directionally solidified as-cast metallic microstructures. However, in contrast to the columnar metallic grains, the PPTA fibrils are generally found to possess a superstructure consisting of small-amplitude (ca. $20 \mathrm{~nm}$ ), high wavelength (ca. 300-500 nm) accordion-style "pleats." The presence of these pleats is often cited as one of the causes for the superior tensile ductility of PPTA fibrils/fibers (e.g., [9]). While intrasheet bonding is of a strong hydrogen-bonding type, intersheet bonding involves weak van der Waals interactions and the formation of $\pi$-type molecular orbitals. Thus, PPTA fibrils possess a substantial level of transverse anisotropy.

As mentioned previously, intersheet bonding within PPTA fibrils is relatively weak, and consequently, fibrils tend to possess various crystallographic and topological intersheet defects (e.g., local delaminations [10]). This is the reason that PPTA fibrils tend to buckle during axial and lateral (sheet-edge-on) compressive loading and to possess relatively inferior axial/transverse compressive properties.

1.1.5. PPTA Fibers. As mentioned previously, PPTA fibers are essentially assemblies of parallel fibrils (nearly coaxial with the fiber itself). While it is generally believed that fibril bundles within the fiber are held together by weak van der Waals or Coulomb forces, it is possible that chain crosslinking and the presence of tie fibrils can play a role in the formation of fibril bundles. A schematic of the basic architecture of the single PPTA fiber and its relationship to the constituent fibrils is depicted in Figure 2. Examination of this figure reveals that the fibril bundles form the fiber core and that the fibers also contain a relatively thin skin region. While this region is comprised of highly oriented PPTA chains, it is usually of a noncrystalline character.

\subsection{PPTA Microstructural Hierarchy}

1.2.1. Structural/Behavioral Complexity of Protective Systems. The work presented in this paper is directly concerned with PPTA single fibers, or more specifically, with the axial compressive response of these fibers. However, the broader goal of the present work is to help improve fidelity and accuracy of the computational models for PPTA-fiberreinforced polymer-metal composites used in the aforementioned blast-/ballistic-protection systems. These protective systems generally possess complex morphology and behavior [5-8], which is mainly attributed to (a) their hierarchical, multilength-scale architecture; (b) their nonlinear and pathand rate-/time-dependent mechanical response; and (c) the operation of various phenomena/processes (e.g., fibril/fiber damage, highly localized deformation and the associated formation of kink/deformation bands, filament twisting, interfilament friction and sliding, etc.).

1.2.2. Major Length Scales. By carrying out a detailed analysis of the structural hierarchy of the PPTA-fiber-reinforced polymer-matrix composite protective structures, eight distinct length scales were identified in our recent work $[5,6]$. The microstructures/architectures of each of these length scales are schematically depicted in Figures 3(a)-3(h). In each case, labels are used to identify the main microstructural constituents. Since a detailed description of these length scales can be found in $[5,6]$, they will not be repeated here. 


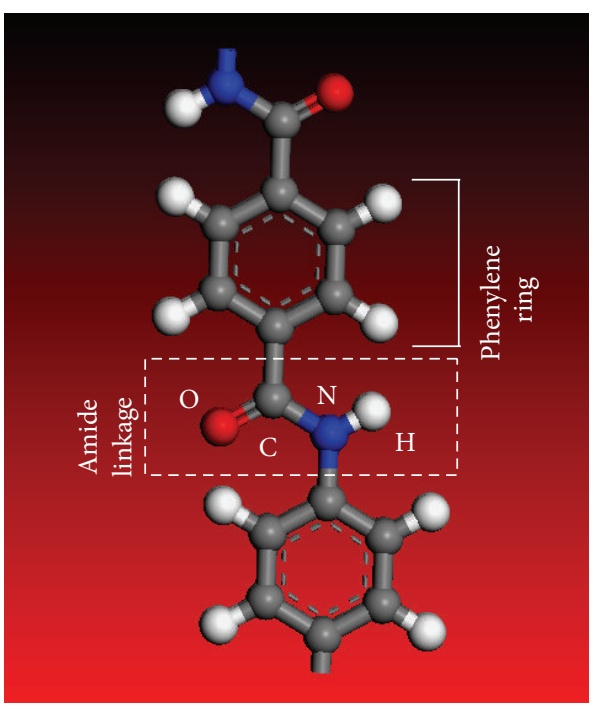

(a)

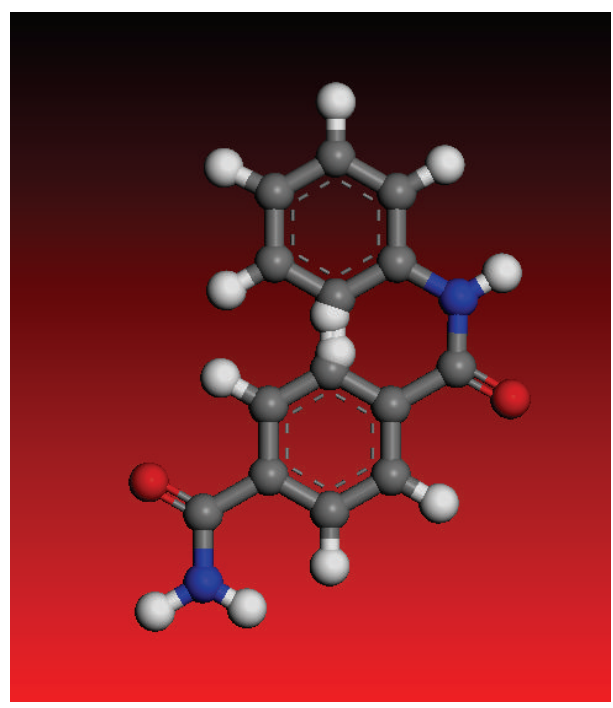

(b)

Figure 1: (a) Trans- and (b) cis-molecular conformations in typical PPTA-based polymeric-material chains/molecules.

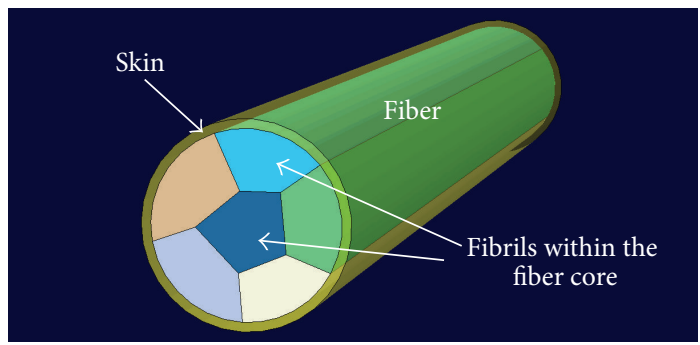

Figure 2: Topological relationship between a single fiber and its constituent fibrils.

\subsection{Material Models for PPTA Fiber-Reinforced Polymer-Matrix Composites}

1.3.1. CAE-Based Design/Development of Protective Systems. Ballistic/blast protection systems based on PPTA fibers have traditionally been designed and developed using legacy knowledge, empiricism, and extensive "fabricate-and-test" procedures. Given the higher costs and often significantly longer lead times associated with this approach, it has gradually been complemented (and partly replaced) by the appropriate cost- and time-efficient CAE analyses. Recent developments in the numerical modeling of transient nonlinear dynamics phenomena, such as those accompanying blast- and ballistic-loading conditions (e.g., [11, 12]) have further accelerated the use of CAE analyses in the design and development of protective structures. However, the accuracy of these CAE-based results depends on the fidelity of the constitutive models for the PPTA-based materials used in constructing protective structures (e.g., [13]). As demonstrated in our recent work [7], the PPTA material models generally used suffer from a number of deficiencies. These deficiencies are associated with (a) inability of the models to fully account for the material response to high-deformationrate, large-strain, high-pressure loading conditions (typically encountered during projectile- or blast-impact events); (b) disregard for the effects of prior treatment/handling of the constituent fiber materials on the resulting material properties; and (c) a lack of inclusion of the changes in the material properties brought about by the protective-structure manufacturing process chain.

1.3.2. Recent Improvements in PPTA Material Model. In the same work [7], it was demonstrated that the first step towards removing these deficiencies is to identify and characterize key phenomena and processes, occurring at different length/time scales and to quantify their contributions to the overall behavior/properties of the subject (continuum type) material. In this regard, the PPTA material-constitutive models were improved in our recent work [5-8] as follows: (a) inclusion of the effect of various fiber- and subfiber-length-scale microstructural features (including the defect structure) on the material dynamic response $[5,6]$; (b) incorporation of the inelastic deformation effects associated with transverse loading and their coupling to the longitudinal mechanical properties of the PPTA fibers [8]; and (c) accounting for the effects of prior longitudinal-compressive and/or axialtorsional loading on the tensile behavior of the PPTA fibers and so accounting for the effect of fiber handling during PPTA-structure fabrication [14].

1.3.3. Proposed Additional Improvements. In the present work, an attempt is made to further improve the PPTAmaterial model used in the CAE analyses. Specific improvements deal with the contribution of the axial compressive response of single PPTA fibers, which results in the formation and propagation of kink bands (fiber-surface irregularities in the form of disclinations/protrusions), Figure 4. Since 


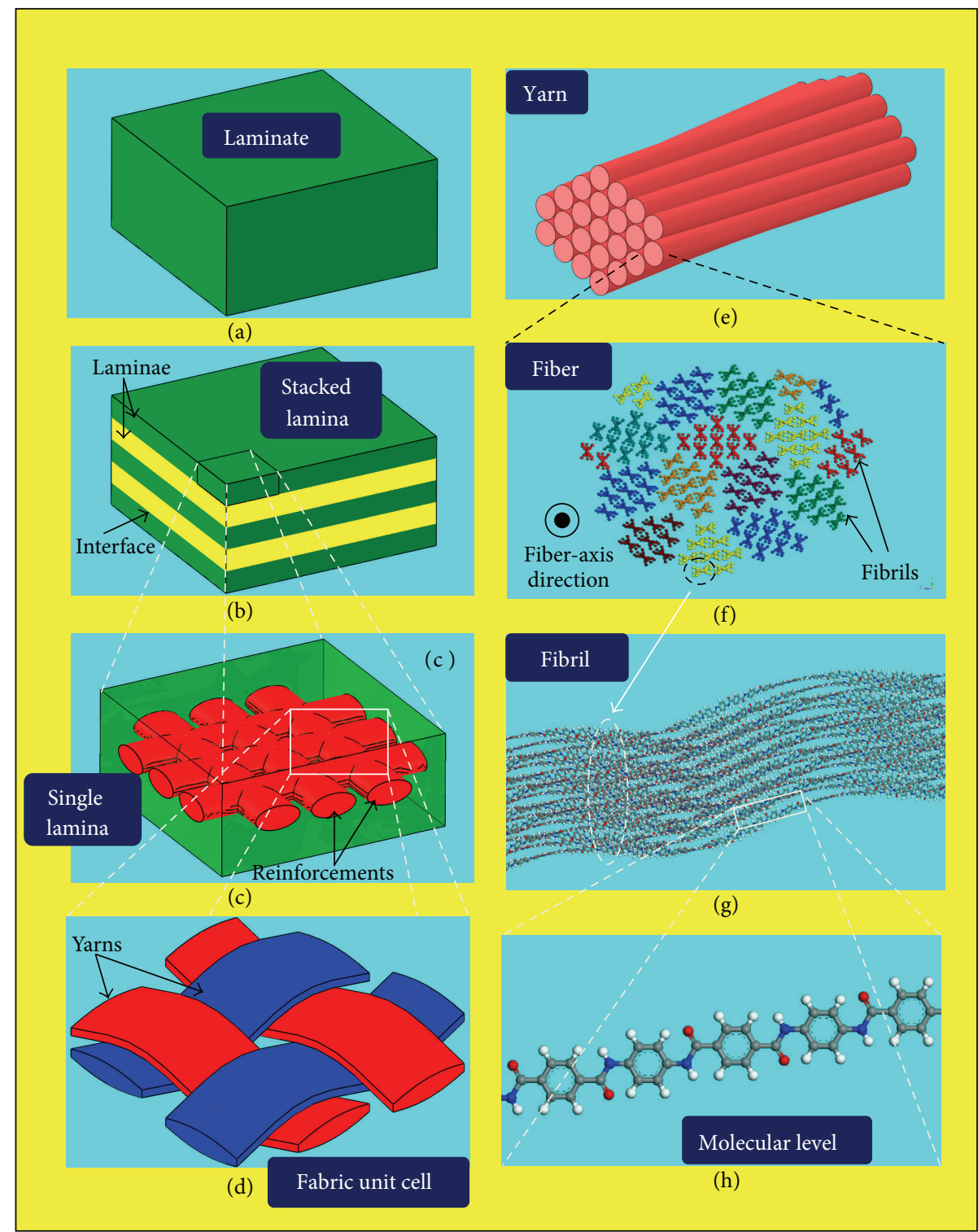

FIGURE 3: Microstructural hierarchy consisting of eight distinct length scales encountered in fiber-reinforced polymer-matrix composites.

PPTA fibers can experience axial-compression (including the one accompanying fiber bending) during fabrication of the reinforcing substructures (e.g., during textile manufacturing of ballistic fabric), the PPTA fiber-reinforced polymermatrix composites and the associated protective structures typically contain kinked fibers (fibers with inferior mechanical properties). In addition, snap-back of elastic unloading following fracture of the stretched fibers (the process which often accompanies ballistic-/blast-impact loading) is often accompanied by the formation of a series of kink bands. The resulting inferior mechanical properties of the kinked fibers can seriously compromise multihit performance of the protective structures.

1.4. Main Objective. The main objective of the present study is to extend our prior molecular-type simulation work [5$8,14]$ in order to (i) provide insight into the phenomena

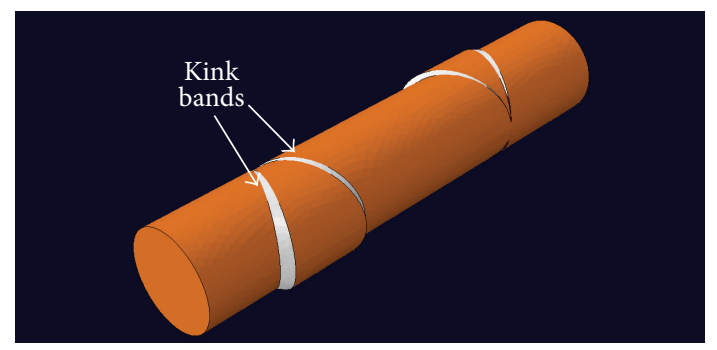

FIGURE 4: A schematic of a series of kink bands formed over a short segment of a PPTA fiber.

and processes associated with the formation and propagation of kink bands within single PPTA fibers during axial compression. Presently, it is not clear if kink bands are merely the result of highly localized crystallographic slip within the 
crystalline/paracrystalline fibrils or are the product of flowinduced chain/fibril buckling/localized bending (generally referred to as "fibrillation") [15]; and (ii) identify the ways and means for passing the information regarding the operation of (discrete) fiber length-scale phenomena and processes (e.g., formation and propagation of kink bands) to the (continuum) lamina/laminate length scales.

It should be noted that while the majority of the ballistic/blast protective systems fabricated today are based on polymer-matrix composites containing Kevlar KM2 fiberbased reinforcing substructures (e.g., fabric mats), the subject of the present work are generic PPTA fibers. This should not be considered as a major simplification since the behavior and properties of various Kevlar and Twaron grades of fibers are generally found to be dominated by their main PPTA constituent. Hence, the inclusion of fiber-gradespecific chemical and molecular-microstructure details was judged unnecessary (in particular, considering the associated additional computational cost).

\section{Effect of Prior Compression on Fiber Tensile Strength}

As mentioned earlier, kink bands resulting from the application of a prior axial/longitudinal compressive loading can negatively affect axial-tensile strength of PPTA fibers. Examination of the open-domain literature revealed two investigations which are particularly pertinent to the present work. A brief overview of these prior investigations is provided in the remainder of this section.

2.1. Dobb et al., 1981 [10]. Dobb et al. [10] carried out longitudinal compression tests on single PPTA fibers and examined the fiber microstructure before and after testing in order to identify microstructural changes responsible for the inferior axial-compressive strength of the fibers. The main findings of Dobb et al. can be summarized as follows: (a) the inferior axial compressive strength of single PPTA fibers is a mere consequence of the formation of kink bands and subsequent fiber buckling; (b) the aforementioned fibril pleats appear not to play a major role in the onset of kink band formation or in band propagation; (c) formation of kink bands has been observed to be closely linked with relatively weak van der Waals-type intersheet bonding, and the associated weak lateral support experienced by the PPTA sheets. These conditions can lead to the phenomena such as localized intersheet shear, sheet-delamination and sheet/chain buckling, all of which appear to stimulate the formation of kink bands; and (d) the presence of the crystalline structure within the PPTA fibrils promotes the formation of the kink bands through the coordinated motion (accompanying highly localized crystallographic slip) of the well-organized PPTA chain segments.

2.2. Deteresa et al., 1984 [16]. Deteresa et al. [16] carried out a systematic experimental investigation of the effect of prior axial compression on the longitudinal tensile strength of single PPTA fibers. The mechanical tests were supplemented by optical and scanning electron microscopy investigations in order to relate changes in the fiber microstructure induced by prior (longitudinal-compressive) deformation to the observed losses in the fiber longitudinal-tensile strength. The results obtained by DeTeresa et al. can be summarized as follows: (a) prior longitudinal compression produces regularly spaced kink bands in the single PPTA fibers; (b) the presence of these bands within a single fiber reduces its longitudinal-tensile strength. Typically, a loss of ca. $10 \%$ in the fiber longitudinal-tensile strength is associated with the prior application of $3 \%$ axial-compressive strain; and (c) axial compression tends to induce well-defined discrete kink bands, suggesting the dominant role of crystallographic slip. This behavior is in sharp contrast to the effect of prior torsional deformation, which tends to induce diffuse shear bands and fibrillation.

\section{Coarse-Grained Fiber-Scale Computational Analysis}

As mentioned earlier, coarse-grained fiber-scale computational methods have been employed in the present work in order to (a) provide insight into the phenomena and processes associated with the formation and propagation of kink bands within single PPTA fibers during axial compression and (b) assess the role of crystallographic/topological defects in the kink band formation process and in the relative importance of localized crystallographic slip and fibrillation processes.

Within the coarse-grained computational framework, atoms, ions and interatomic bonds are not modeled explicitly, but rather, individual atoms/ions are combined into larger single particles, referred to as "beads," where each bead represents the collective degrees of freedom of the constituent atoms/ions. Neighboring beads residing within the same chain are then connected using bead/bead bonds (also referred to as connectors), while nonbonded beads are allowed to interact via van der Waals-like and Coulomb-like force fields (the term "force field" will be defined later). Since the coarse-grained computational framework employs larger particles (relative to the particle sizes encountered in the so-called all-atom computational analyses), larger computational domains can be analyzed (simulating the maximum number of particles permitted by the available computational and storage resources). In addition, by integrating out the high-frequency vibrations of the constituent atoms/ions and allowing the system dynamics to be controlled by the compliant/soft, bead/bead interactions (rather than by stiff/hard, atom/atom interactions), a significantly longer time scale is attained (relative to that which could be afforded by the allatom computational method).

It should be noted that while only coarse-grained molecular methods and tools are used in the present work, the results of the all-atom molecular dynamics simulations carried out in our recent work $[5-8,14]$ were utilized. Specifically, all-atom molecular dynamics calculations described in $[5-8,14]$ were used to (a) identify the nature and the number of types of 
coarse-grained beads; (b) for each bead type to quantify the bead mass and bead size; and (c) help parameterize intra- and interchain coarse-grained force-fields (a set of mathematical expressions describing various contributions to the potential energy function for the system of interacting particles, that is, beads, in the present case). A brief description of the all-atom computational procedures employed in our recent work [5$8,14]$ and of the associated results used in the present work is provided in the following.

3.1. PPTA Coarse-Graining and Force-Field Parameterization. Within the all-atom computational methods, molecularlevel models of individual PPTA chains based on the trans molecular conformation of the repeat unit, Figure 1(a), are constructed using the Visualizer [17] program from Accelrys. Typically, PPTA chains consisting of 20 repeat units and having the molecular weight of ca. $4760 \mathrm{Da}$ are used.

3.1.1. PPTA Coarse Graining. Since PPTA can be considered as a homopolymeric material and in order to be able to handle computational domains of a larger size, only one type of bead is introduced during the coarse-graining procedure of the PPTA chains. Consequently, the bead mass was set equal to that of the PPTA repeat unit while the volume allotted to each bead was readily derived from the PPTA-fiber mass density.

3.1.2. Coarse-Grained Force-Field Functions. The coarsegrained force field used in the present work includes four (functionally distinct) contributions to the system potential energy (a) intrachain bond-stretch term; (b) interchain twobond-angle term; (c) intra-sheet hydrogen-bond term; and (d) intersheet van der Waals nonbond interaction term. The functional forms for the four potential-energy terms are given as follows.

IntraChain Bond-Stretch Term. Covalent bonding within the bead type PPTA chains is represented using the following form of a harmonic-type energy function:

$$
E^{\text {stretch }}=\frac{1}{2} K^{\text {stretch }}\left(r-r_{o}^{\text {stretch }}\right)^{2}
$$

where $K^{\text {stretch }}$ denotes the bond-stiffness parameter, $r$ the connector (i.e., bead bond) length, and the subscript $o$ is used to denote the equilibrium value of the associated quantity. Due to the aforementioned high stretching stiffness of the PPTA molecules, $K^{\text {stretch }}$ is assigned a relatively large value, as will be shown later.

IntraChain Two-Bond-Angle Term. An analogous harmonic function is used to represent the potential energy contribution associated with the intrachain two-bond-angle term as

$$
E^{\text {angle }}=\frac{1}{2} K^{\text {angle }}\left(\theta-\theta_{o}^{\text {angle }}\right)^{2}
$$

where $K^{\text {angle }}$ denotes the bond-stiffness parameter and $\theta$ the intrachain interbond angle. Due to the aforementioned high bending rigidity of the PPTA molecules, $\theta_{o}^{\text {angle }}$ is set to a value of $\pi$, while, as will be shown later, $K^{\text {angle }}$ is assigned a relatively large value.

Intra-Sheet Hydrogen-Bond Term. Following our prior work on polyurea [18-20], the potential energy associated with intra-sheet interchain hydrogen bonding is expressed using the 18-9 Lennard-Jones type of function as

$$
E^{\text {hydrogen }}=\varepsilon^{\text {hydrogen }}\left[\left(\frac{r_{o}^{\text {hydrogen }}}{r}\right)^{18}-2\left(\frac{r_{o}^{\text {hydrogen }}}{r}\right)^{9}\right] \text {, }
$$

where $-\varepsilon^{\text {hydrogen }}$ is the interaction energy minimum and represents the strength of hydrogen-bond interaction between two beads, while $r_{o}^{\text {hydrogen }}$ is the associated hydrogen-bonded interbead equilibrium distance.

InterSheet van der Waals Nonbond Interaction Term. As far as the non-bond van der Waals interaction energy involving beads residing on different PPTA sheets is concerned, it is represented using the 12-6 Lennard-Jones type of function as:

$$
E^{\text {non-bond }}=\varepsilon^{\text {non-bond }}\left[\left(\frac{r_{o}^{\text {non-bond }}}{r}\right)^{12}-2\left(\frac{r_{o}^{\text {non-bond }}}{r}\right)^{6}\right] \text {, }
$$

where $-\varepsilon^{\text {non-bond }}$ is the interaction energy minimum and represents the strength of nonbond interaction between two beads, while $r_{o}^{\text {non-bond }}$ is the associated nonbonded interbead equilibrium distance.

3.1.3. Coarse-Grained Force-Field Parameterization. Procedures used for parameterization of each of the four parts of the coarse-grained force field are presented in this section.

IntraChain Bond-Stretch Term. Since the coarse-grained formulation used in the present work defines only one type of bead, the bond stretch force-field term contains only two parameters: $K^{\text {stretch }}$ and $r_{o}^{\text {stretch }} \cdot r_{o}^{\text {stretch }}$ was determined by setting it equal to the equilibrium bead/bead separation, as computed using all-atom molecular statics calculations of the minimum potential-energy configuration of a single infinitely long, perfectly straight PPTA chain.

To determine $K^{\text {stretch }}$, an equilibrium molecular-dynamics-based free-energy calculation procedure is combined with the so-called "Adaptive Biasing Force" (ABF) method [19]. During the application of the ABF method, the distance between the centers of mass of the (all-atom represented) beads is varied in small increments from its initial (minimum energy) value by appropriately changing the relative position of all constituent atoms. This was followed by prolonged ( $40 \mathrm{~ns}$ simulation time, at a temperature of $300 \mathrm{~K}$ ) all-atom equilibrium molecular dynamics simulations. The trajectory results obtained, averaged over the simulation time, are used to compute the mean force acting on the beads, quantifying the tendency of the beads' connector 
to recover its equilibrium length. To cancel this force, a biasing force of equal magnitude and opposite sign is equally partitioned and applied to all the constituent atoms in the two beads. This leaves the system under zero mean force and only the fluctuating part of the force remains, enabling the computation of the "equilibrium" thermodynamic properties of the system, for example, mean potential energy at a given value of the bead connector length. When the resulting potential energy versus connector length results are fitted using linear regression analysis in conjunction with (1), the sought-after stretching stiffness parameter is obtained. The resulting functional relationship for bond-stretch energy versus bonded-bead separation is depicted in Figure 5(a).

IntraChain Two-Bond-Angle Term. The two-bond-angle force-field term also contains only two parameters: $K^{\text {angle }}$ and $\theta_{o}^{\text {angle }}$. As mentioned earlier, $\theta_{o}^{\text {angle }}$ is set to a value of $\pi$. As far as the bending stiffness parameter $K^{\text {angle }}$ is concerned, it is determined using an ABF-based-procedure analogous to that described for the case of $K^{\text {stretch }}$. The main difference is that instead of a perfectly straight PPTA molecule, a molecule consisting of two perfectly straight halves with an included angle $\theta$ (of varying magnitude) is utilized. The resulting functional relationship for bond-angle energy versus two-bond-angle is depicted in Figure 5(b).

Intra-Sheet Hydrogen-Bond Term. Due to the presence of a single bead type, the hydrogen-bond part of the coarsegrained force field is also characterized by two parameters: $\varepsilon^{\text {hydrogen }}$ and $r_{o}^{\text {hydrogen }}$. These two parameters were determined using the same procedure as the ones described above, except that instead of a single infinitely long, perfectly-straight chain, a wall of parallel, equally spaced, infinitely-long, perfectly straight chains is used, and the interchain spacing is varied. The resulting functional relationship for hydrogenbond energy versus intra-sheet interchain separation distance is depicted in Figure 5(c).

InterSheet van der Waals Non-Bond Interaction Term. The two non-bond interaction parameters $\varepsilon^{\text {non-bond }}$ and $r_{o}^{\text {non-bond }}$ are determined using the same procedure, except that an infinitely wide/deep/tall stack of PPTA sheets (with the ABABAB. . . stacking), each consisting of an array of parallel, equally spaced, infinitely long, perfectly straight chains, is used. The resulting functional relationship for nonbond energy versus intersheet separation distance is depicted in Figure 5(d).

Parameterization Summary. A summary of the eight coarsegrained PPTA force-field parameters is provided in Table 1.

3.2. Coarse-Grained Molecular Method. All-atom and coarsegrained molecular methods and tools are fundamentally identical, since both treat materials as assemblies of interacting particles and use force fields to quantify various bonding and nonbonding interactions. The two main differences between the two methods are (a) the nature and size/mass of the constituent interacting (atoms versus beads) particles;
TABLE 1: Coarse-grained PPTA force-field parameterization used in the present work. Please see (1)-(4) for explanation of the symbols.

\begin{tabular}{lcc}
\hline Parameter & Unit & Value \\
\hline$K^{\text {stretch }}$ & $\mathrm{kJ} / \mathrm{mol} \cdot \mathrm{nm}^{2}$ & 6831.1 \\
$r_{o}^{\text {stretch }}$ & $\mathrm{nm}$ & 1.31 \\
$K^{\text {angle }}$ & $\mathrm{kJ} / \mathrm{mol} \cdot \mathrm{rad}^{2}$ & 9,752 \\
$\theta_{o}^{\text {angle }}$ & $\mathrm{rad}$ & $\pi$ \\
$\varepsilon^{\text {hydrogen }}$ & $\mathrm{kJ} / \mathrm{mol}$ & 22.3 \\
$r_{o}^{\text {hydrogen }}$ & $\mathrm{nm}$ & 1.39 \\
$\varepsilon^{\text {non-bond }}$ & $\mathrm{kJ} / \mathrm{mol}$ & 11.1 \\
$r_{o}^{\text {non-bond }}$ & $\mathrm{nm}$ & 1.52 \\
\hline
\end{tabular}

and (b) bead/bead interactions are generally quite more compliant (softer) than their atom/atom counterparts.

In general, a complete formulation of a coarse-grained molecular simulation problem includes, at a minimum, the following four items: (a) a computational model consisting of bead-type chains and larger structures (e.g., sheets, fibrils, fibers, etc.); (b) a set of interbead force-field potentials which describe accurately various bonding and nonbonding bead/bead interactions; (c) a computational method(s) to be used in the simulations; and (d) formulation of the problem to be analyzed. These four items are discussed in greater detail in the remainder of this section.

3.2.1. Computational Model(s). While the coarse-grained computational approach allows a substantial increase in the dimensions of the computational cell analyzed, a coarsegrained analysis of a segment of the PPTA fiber (typical diameter on the order of $10 \mu \mathrm{m}$ ) would still require the use of a prohibitively large number of beads. Considering the fact that the majority of the crystalline/topological defects are located in the core of the PPTA fibers and that these defects play an important role in the kink-band nucleation process, the computational domain used focuses on the core section of the PPTA fibers. Furthermore, since the transverse cross-section of the computational domain is still small in comparison to that of the PPTA-fiber core, the computational domain is assumed to extend indefinitely in the lateral directions (as well as in the axial direction). The rectangular-parallelepipedshaped computational cell used in the present work is shown in Figure 6(a). The long edge, $c$, of the computational cell is aligned with the fiber axis (which is, in turn, aligned with the $z$-coordinate axis). The remaining two transverse edges $(a$ and $b)$ of the cell are aligned, respectively, with the coordinate axes $x$ and $y$. Typically, computational cells of the size $a=24.3 \mathrm{~nm}, b=24.3 \mathrm{~nm}$, and $c=28.6 \mathrm{~nm}$ are used. The infinite extension of the computational domain in the three orthogonal directions is achieved by applying the periodic boundary conditions across the computational cell faces.

Examination of the transverse cross-section of the computational cell shows that the cell consists of nine coaxial fibrils (identified by different colors). While each fibril contains the same PPTA crystal structure, the transverse orientation of this structure differs among the fibrils. However, in all fibrils, the orientation of the crystal lattice is such that the 


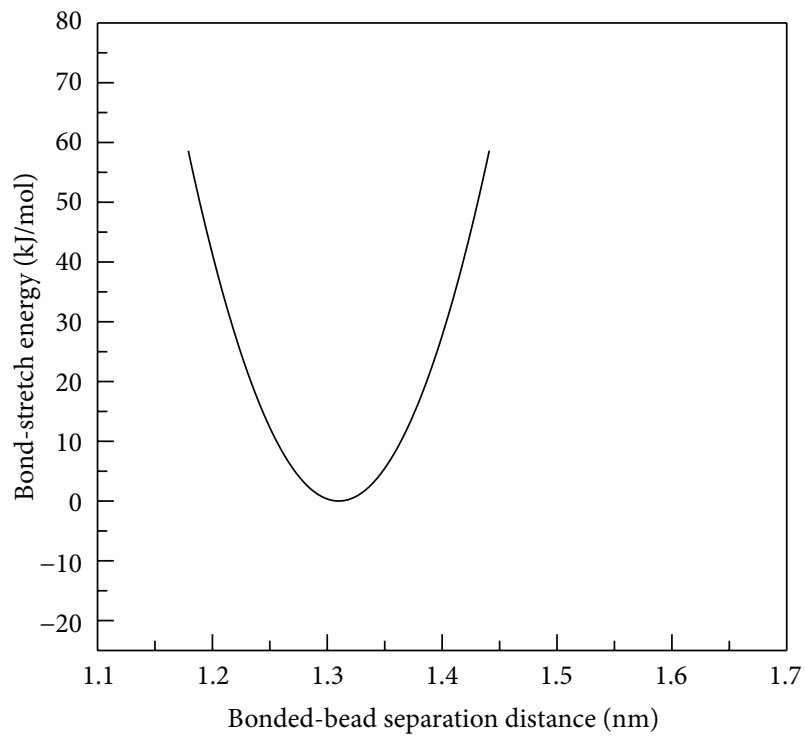

(a)

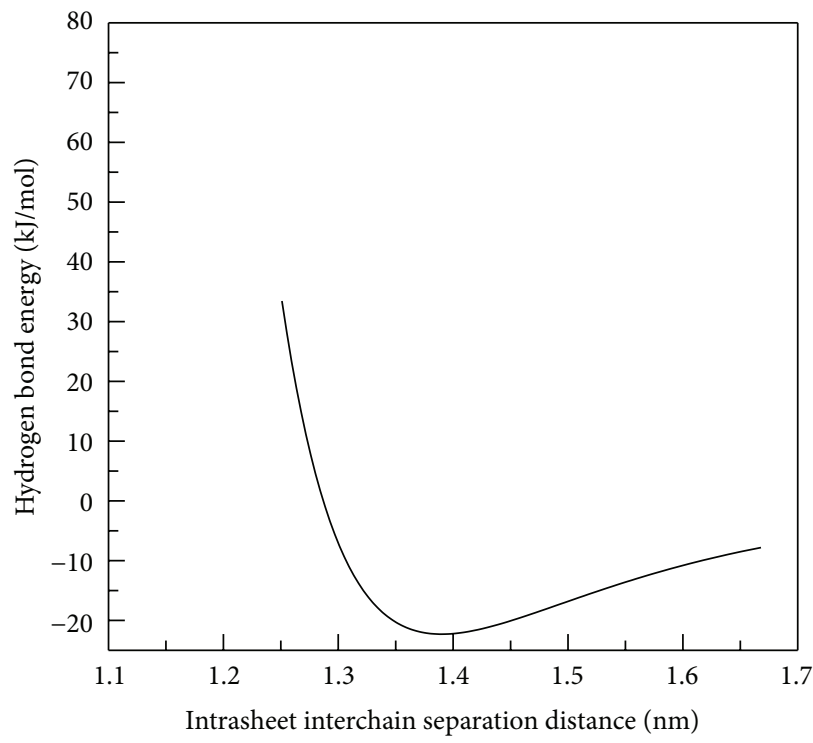

(c)

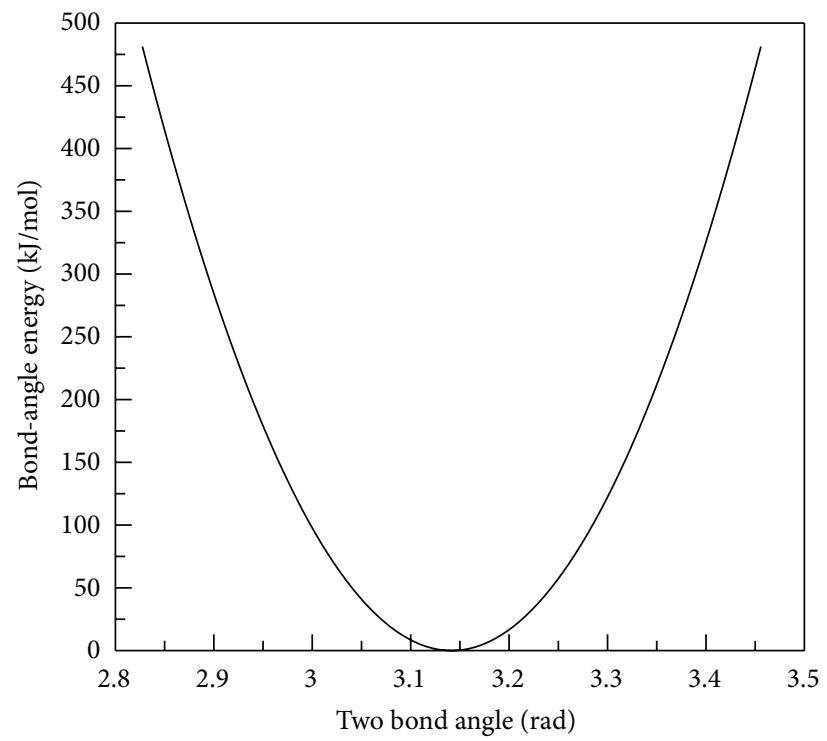

(b)

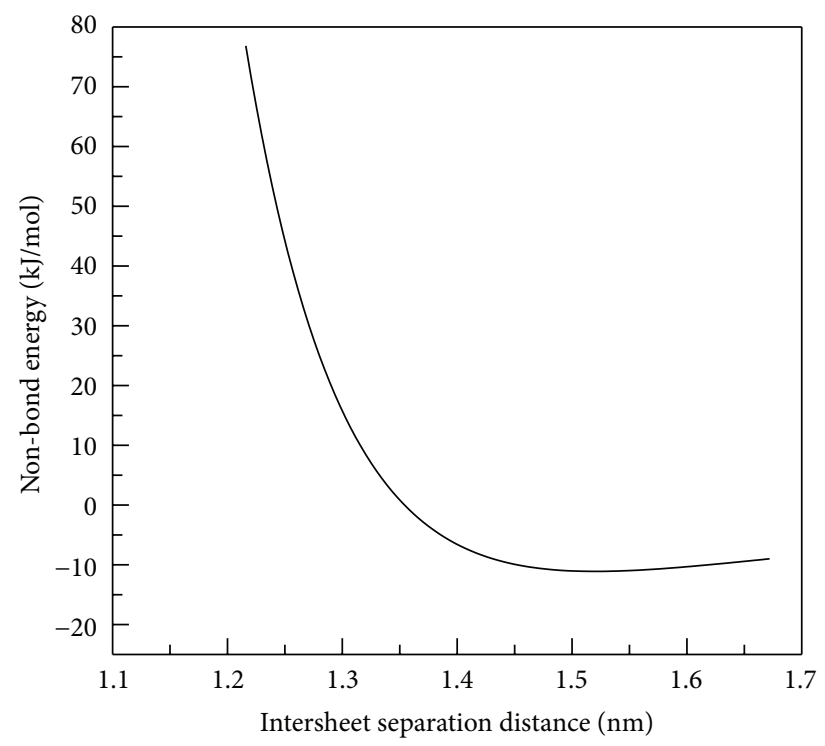

(d)

FIGURE 5: Coarse-grained PPTA force-field functions used in the present work: (a) bond-stretch term; (b) two-bond-angle term; (c) hydrogenbond term; and (d) non-bond term.

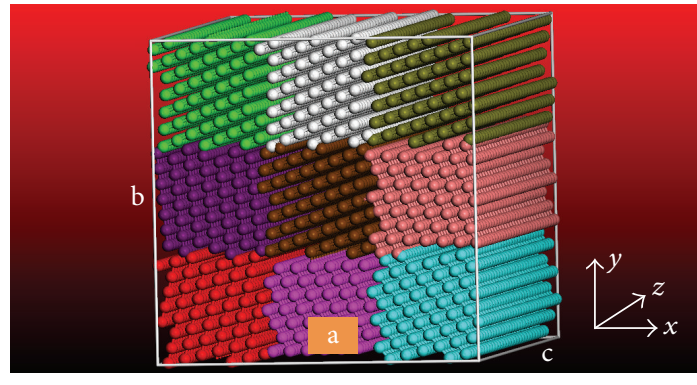

(a)

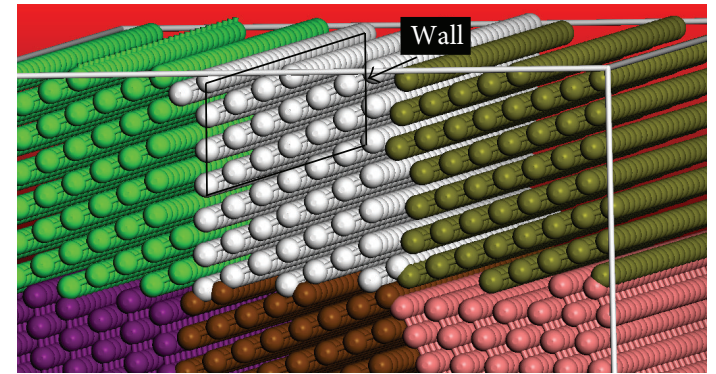

(b)

FiguRE 6: Coarse-grained PPTA fiber computational cell consisting of nine coaxial fibrils (indicated using different colors): (a) zoomed-out view; and (b) a close-up view. 
sheet normal is orthogonal to the fiber axis. The mean massdensity within each fibril is initially set to be the same (by properly adjusting interchain distances within the sheets and intersheet distances) for each fibril. Before conducting any analysis/testing of the computational cell, the potential energy of the computational cell is minimized with respect to the spatial coordinates of the constituent beads. It should be noted that the atomic configuration displayed in Figure 6(a) is the initial (before energy minimization) configuration. A close up of this configuration is displayed in Figure 6(b). This figure clearly shows the formation of PPTA sheets which contain covalent intrachain bonds and interchain, intrasheet hydrogen bonds (both depicted using the ball-and-stick representation).

3.2.2. Force Fields. The force-field functions used, the procedure employed for their parameterization and the resulting parameters' identification were all covered in Section 3.1 and, hence, will not be repeated here.

3.2.3. Computational Method(s). Within the coarse-grained fiber-scale computational investigation carried out in the present work, two types of computational methods, molecular statics (i.e., potential-energy minimization) and (equilibrium) molecular dynamics (i.e., particle-trajectory analysis) are used. These methods are implemented in Discover (a general-purpose atomistic simulation program) from Accelrys [21], the computational tool which was employed in the present work. Within the molecular statics approach, the potential energy of the subject discrete-particle material system is minimized with respect to the position of the particles/beads. On the other hand, within the molecular dynamics approach, gradient of the potential energy (as defined by the force-field functions) with respect to the particle positions is first used to generate forces acting on the particles, and then, the associated Newton's equations of motion (for all particles) are integrated numerically in order to determine the temporal evolution of the particle positions (i.e., particle trajectories). In addition, within the equilibrium molecular-dynamics methods, the system under consideration is coupled to an (external) environment (a constant temperature reservoir, in the present case) which ensures that the system remains in thermodynamic equilibrium (i.e., the system does not experience fluxes of thermodynamic quantities). In the present work, an NVT (where $N$ is the (fixed) number of atoms, $V$, the computational cell volume (also fixed, and set to an arbitrarily large value to provide for the isolated-beads interaction environment), and $T(=300 \mathrm{~K})$ is the temperature) rendition of the molecular dynamics method is employed.

As will be shown in the following, combined application of the molecular statics and equilibrium molecular dynamics methods enables the investigation of (a) axial-compressive behavior of the PPTA fiber core; (b) formation of kink bands; and (c) elucidation of the role different crystallographic/topological defects play in the process of kink-band formation. All the calculations in the present work were carried out by coupling a user-defined coarse-grained forcefield database with the Discover solver [21]. The procedure employed to simulate PPTA fiber-fiber axial compression is implemented and linked with Discover through the use of an input file written in the BTCL script. This enabled precise control of all simulation tasks such as sequential contraction of the computational cell in the longitudinal direction (during the simulation of the axial-compression test). In a few cases, axial-tension of the fibers which were first subjected to longitudinal compression was investigated and, in this case, loading is also applied through the use of an input file written in the BTCL language.

3.2.4. Problem Formulation. As mentioned earlier, the problem analyzed in the present work involves the mechanical response of PPTA fibers subjected to longitudinalcompression (and, in a few cases, subsequently subjected to longitudinal tension). Typically, PPTA fiber diameter is on the order of $10 \mu \mathrm{m}$ and thus $10^{3}-10^{4}$ times larger than the transverse edge length of the coarse-grained computational cell, analyzed in the present work. As established earlier, the computational cell used represents the material located within the fiber core (the region of the fibers which has the highest probability for the presence of flaws/defects and thus the highest potential for kink-band formation). In a typical coarse-grained axial loading analysis of the PPTA fibers, both the initial compression and, where applicable, subsequent longitudinal tension were applied in small increments and each incremental deformation is followed by the computational cell energy-minimization and extended (ca. $40 \mathrm{~ns}$ ) molecular-dynamics simulation runs.

3.2.5. Postprocessing Data-Reduction Analysis. The coarsegrained fiber-level particle-trajectory computational results obtained are mainly used to reveal the response of single PPTA fibers to uniaxial compression (including the microstructural/topological evolution processes such as kink band formation and fibrillation). Fiber axial compression is simulated by subjecting the unit cell to longitudinal compressive deformation in the $z$-direction while allowing for the operation of the Poisson's effects in the $x$ - and $y$-directions. In this case, except for graphical display, no data postprocessing procedure had to be employed. However, in a few simulations, the effect of these processes on the residual axial-tensile strength of the fiber was investigated. In these cases, (a) following uniaxial compression and the formation of kink bands/fibrillation, the unit cell is subjected to longitudinal tensile deformation while allowing for the operation of the Poisson's effects in the transverse directions; and (b) the trajectory data had to be postprocessed to extract and quantify the effect sought after. During the application of the tensile deformation, the procedure described in our recent work [18] was utilized to compute the normal axial stress and its rate of change increase with an increase in the axial tensile strain. The longitudinal-tensile strength of the single PPTA fibril is then set equal to the value of the corresponding normal stress at which the rate of stress increase with increase in strain begins to decline "appreciably" (denoting the onset of inelastic-deformation and/or damage-initiation processes). 


\section{Results and Discussion}

In this section, the results of the coarse-grained fiber-level computational analyses are used to assess (a) axial compressive response of single PPTA fibers; (b) kink-band formation and fibrillation processes; (c) the effect of prior longitudinal compression on the residual axial tensile strength of single PPTA fibers; and (d) the role of various microstructural and topological defects on the kink-band formation/fibrillation propensity of the PPTA fibers and the effect of these localized deformation/damage processes on the residual longitudinaltensile strength of the fiber.

Since the work presented in this section considers PPTA fibers which are initially either perfectly crystalline or crystalline but contain various defects, fibers which are subjected to tensile loading directly or after being subjected to a preludial axial compression and attention is paid to both defect-induced and compression-induced changes in the material microstructure and the longitudinal tensile strength, the corresponding results are presented and discussed within separate sections (each dealing with a particular case of the fiber crystallinity and prior axial compression).

\subsection{Longitudinal-Tensile Strength of Defect-Free Virgin PPTA} Fibers. The procedure for calculation of the fiber longitudinal-tensile strength was described in Section 3.2. This procedure, in the case of defect-free virgin PPTA, yielded a fairly repeatable value of the fibril longitudinal-tensile strength of approximately $6.3 \mathrm{GPa}$. Close examination of the fiber microstructure at the onset of inelastic deformation/damage initiation revealed that, in this case, the longitudinal-tensile strength is controlled by the onset of pronounced degradation of interfibril bonding as well as interchain, intra-sheet bonding. It should be noted that the term "virgin" is used to denote the (either perfectly crystalline or defective) PPTA fibers which were not subjected to axial compression prior to longitudinal tension.

4.2. Microstructure Evolution during Axial Compression of Defect-Free PPTA Fibers. Evolution of the coarse-grained microstructure, during axial compression, within the computational cell containing (initially) defect-free virgin PPTA fibers, is depicted in Figures $7(\mathrm{a})-7(\mathrm{~d})$. The longitudinal stretch values associated with Figures 7 (a) -7 (d) are (a) 0.975; (b) 0.950 ; (c) 0.925 ; and (d) 0.900. The lateral stretches, in each case, take on the values required by the zerolateral-stress condition. Examination of the results displayed in these figures clearly reveals formation and subsequent growth/thickening of two counter-balancing nonparallel shear bands.

The shear bands formed are found to be of a reversible nature, that is, they vanish upon removal of the axial compressive loading. Detailed examination of the microstructure obtained after removal of the axial-compressive loading revealed that this microstructure is very similar to that observed in the defect-free virgin PPTA fiber. In other words, preludial axial compression in the case of defect-free PPTA fibers does not cause any significant permanent change in the fiber microstructure.
4.3. Longitudinal-Tensile Strength of Defect-Free Precompressed PPTA Fibers. Application of the aforementioned longitudinal-tensile-strength determination procedure to the defect-free precompressed PPTA fibers revealed that this strength is practically identical to its counterpart $(6.3 \mathrm{GPa})$ obtained in the case of defect-free virgin PPTA fibers. This finding is fully consistent with the aforementioned observation that the preludial compression (at least up to the compressive strain of $\ln (0.9)=-0.105)$ does not introduce any significant permanent changes in the fiber material microstructure.

4.4. Microstructure of Defective Virgin PPTA Fibers. In our recent work $[5-8,14]$, a detailed overview was provided with respect to the character of the main microstructural/topological defects found in PPTA fibers, their classification, their origin in relation to the PPTA synthesis/processing conditions, and their (estimated) typical concentrations. Consequently, a similar detailed overview will not be provided here. However, it should be established that among the isolated and clustered point-type defects, the following were identified as having the largest effect on the PPTA-fiber properties (in particular, on the longitudinaltensile strength): (a) chain-ends; (b) side groups; and (c) voids and interstitials. In addition, planar-type defects, commonly referred to as defect bands, were also found to have a major effect on the fiber strength. In the present work, only the effect of (isolated or clustered) chain ends or side groups was investigated. Chain ends are the defects which stem from the fact that some of the fibril chains do not run continuously along the fiber length but rather terminate (at one or both ends) within the fiber. As far as the side-group defects are concerned, they are associated with the presence of radicals, for example, $-\mathrm{SO}_{3} \mathrm{H},-\mathrm{SO}_{3}{ }^{-} \mathrm{Na}^{+}$, between the PPTA sheets. As shown in our previous work $[5-8,14]$, the presence of these two types of defects and their clusters within the (otherwise) perfect PPTA fibers causes local changes in the material microstructure. These microstructural changes, as will be shown in the following, affect the fiber longitudinal-tensile strength.

4.5. Longitudinal-Tensile Strength of Defective Virgin PPTA Fibers. Application of the longitudinal-tensile-strength determination procedure clearly revealed that the fiber strength is highly sensitive to the presence, type, and cluster size of the two types of defects analyzed. Due to the stochastic nature of the spatial distribution of the defect concentration within the fiber, the longitudinal-tensile strength of the fibers has also been found to be a stochastic quantity. This finding is in full agreement with several experimental reports (e.g., [2224]). In our recent work [6], a procedure was introduced for the derivation and parameterization of a probability density function for the longitudinal-tensile strength of PPTA fibers. This procedure will be used in the present work (without reviewing the details). When applying this procedure, (isolated and clustered) defect concentrations were set to their values associated with the prototypical PPTA fiber fabrication conditions [6]. 


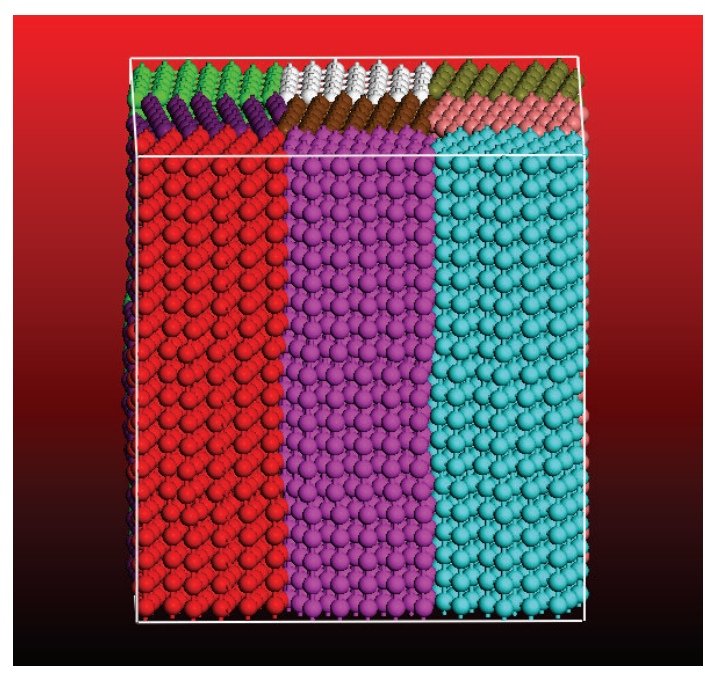

(a)

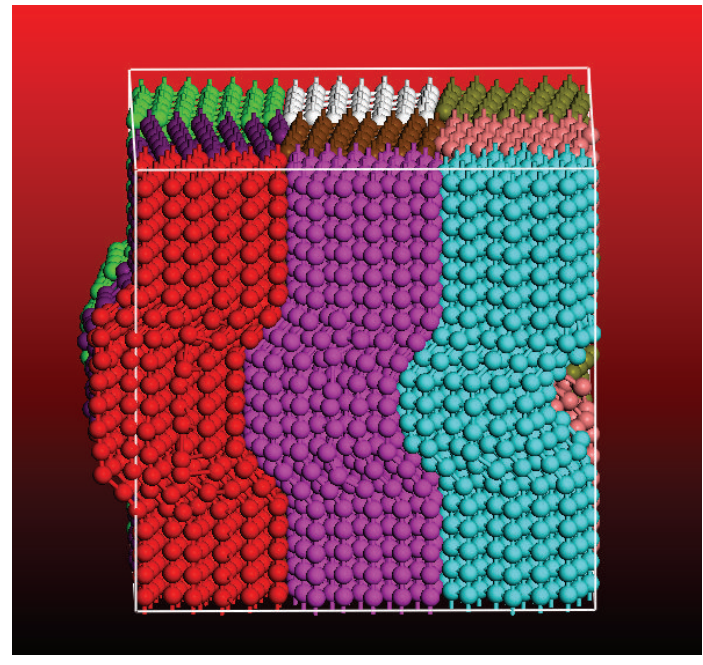

(c)

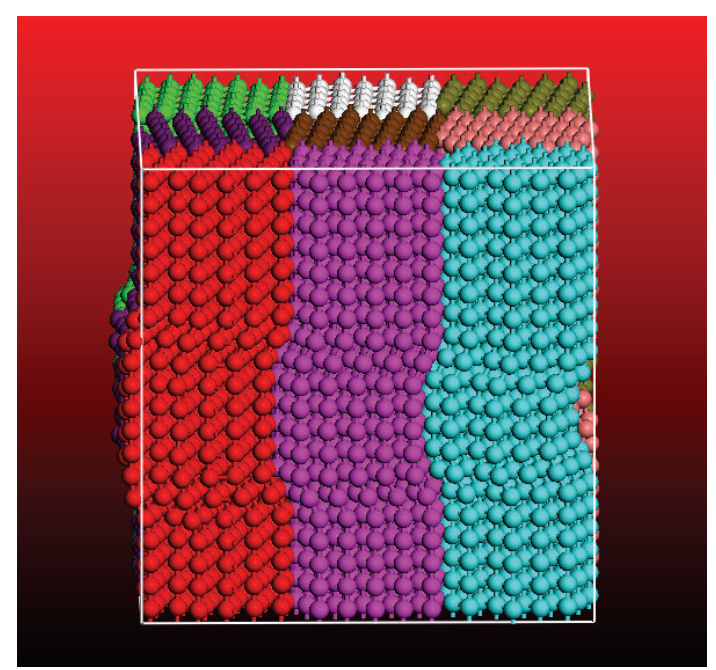

(b)

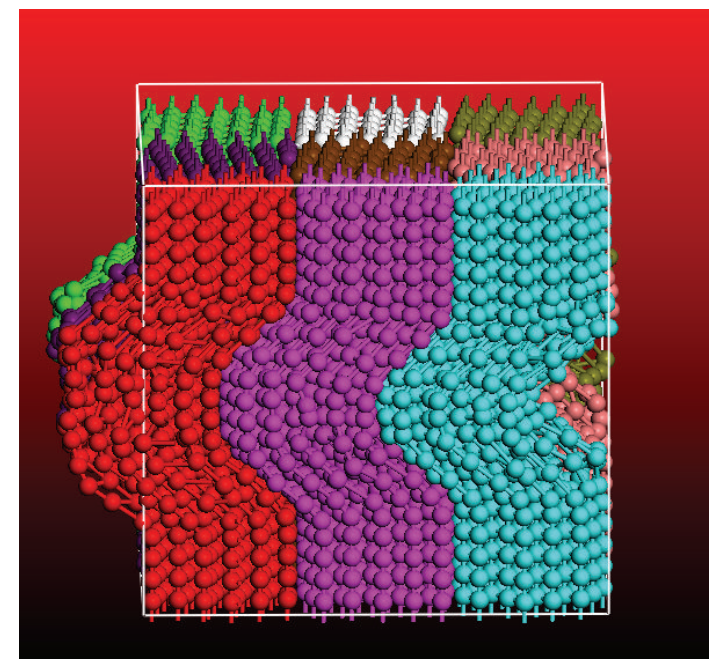

(d)

FiguRE 7: Coarse-grained microstructure, during axial compression, within the computational cell containing (initially) defect-free virgin PPTA fiber at the longitudinal stretch values of (a) 0.975; (b) 0.950; (c) 0.925 ; and (d) 0.900 .

Application of this procedure to the results obtained in the present work yielded the virgin-defective-crystal PPTA-fiber longitudinal-tensile-strength probability density and (complementary) cumulative density functions depicted, respectively, in Figures 8(a) and 8(b). It should be noted that the probability density function in Figure $8(a)$ is represented by its corresponding relative frequency, while the complementary cumulative density function displayed in Figure 8(b) is obtained by integrating the relative frequency from $\infty$ to the given value of the fiber axial strength. The mean value of the virgin-defective-PPTA longitudinal tensile strength, computed as the first moment of the probability density function displayed in Figure 8(a), is found to be $~ 5.0 \mathrm{GPa}$. This value is in reasonably good agreement with the measured PPTA-fiber longitudinal-tensile strength (typically found to be in a $3-5 \mathrm{GPa}$ range (e.g., $[20,23,25,26])$. This finding suggests that the present computational procedure can reasonably well account for the experimental observations regarding the PPTA fiber longitudinal-tensile strength. The cumulative distribution displayed in Figure 8(b) defines the probability that the PPTA fiber longitudinal-tensile strength is not lower than a given (lower bound) value, and this type of function is often used in probabilistic/reliability-based design analyses [27]. In such analyses, functional constraints cannot be met with absolute certainty but rather with an acceptably low level of failure.

4.6. Microstructure Evolution during Axial Compression of Defective PPTA Fibers. Introduction of chain-end isolated or clustered defects into the PPTA fiber did not change the nature of the microstructure evolution during longitudinal compression. In other words, axial compression was accompanied by the formation and growth of counterbalancing kink bands. However, kink bands are found to occur at a lower level of axial-compressive strain (revealing the stimulating effect that these defects have on the kink-band 


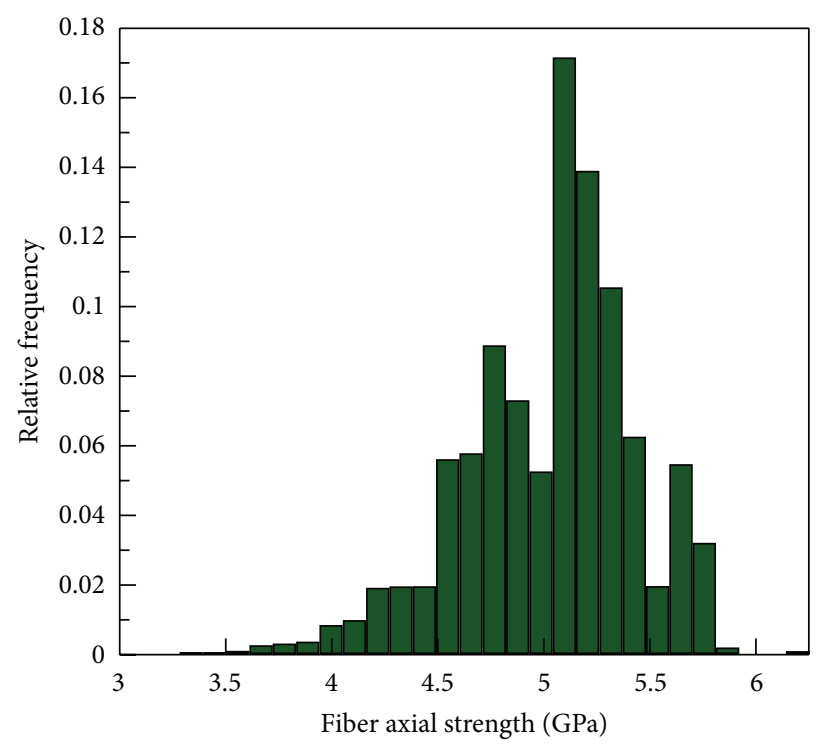

(a)

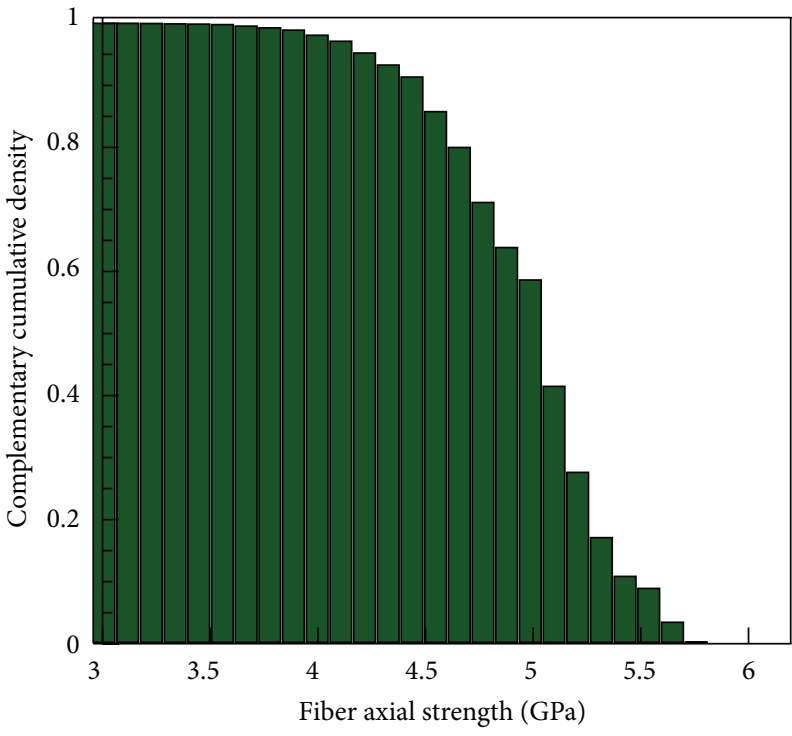

(b)

FIGURE 8: (a) Longitudinal-tensile-strength relative frequency (i.e., probability density function); and (b) the corresponding complementary cumulative density function for defective virgin PPTA-fibers.

formation). Since the fiber microstructures, at different stages of axial compression, obtained in this case are very similar to those displayed in Figures 7(a)-7(d), they are not shown here. Another difference between the axial-compressive behavior of the defect-free and the defective PPTA fibers is that, in some cases, removal of the axial-compressive loading did not result in complete disappearance of the kink bands.

When isolated or clustered side-group defects were introduced into the PPTA fiber, axial compression was not accompanied by the formation of well-defined kink bands. Rather, microstructural changes resembling fibrillations were observed $[28,29]$. An example of the microstructural evolution during axial compression of PPTA fibers containing sidegroup defects is depicted in Figures 9(a)-9(d). The longitudinal stretch values associated with Figures 9(a)-9(d) are (a) 0.975 ; (b) 0.950 ; (c) 0.925 ; and (d) 0.900 . The lateral stretches, in each case, take on the values required by the zero-lateralstress condition. Examination of the results displayed in these figures reveals the formation of bulges (an indication of the operation of fibrillation processes) at the computational-cell lateral faces. The height and the extent of these bulges increase in the course of axial compression. In most cases analyzed, microstructural changes experienced, during preludial compression, by the PPTA fiber containing side-group defects were permanent. That is, with the exception of minor elasticrelaxation processes, removal of the compressive loading did not restore the original/uncompressed microstructure.

4.7. Longitudinal-Tensile Strength of Defective Precompressed PPTA Fibers. In the case of PPTA fibers containing chain-end defects, application of the prior axial compression was found not to measurably change the longitudinal-tensile-strength probability density and cumulative density functions (the results not shown for brevity) relative to their counterparts observed in the uncompressed PPTA fibers containing the same type/number of defects. This finding is fully consistent with the fact that, in most cases, kink bands formed during axial compression of the PPTA fibers containing chain-end defects vanish upon the removal of the compressive loading with no major residual changes in the fiber microstructure.

In sharp contrast to the finding just described, in the case of PPTA fibers containing side-group defects, preludial axial compression is found to change the longitudinal-tensile strength. Typical results obtained in this case are presented in Figures 10(a), and 10(b). The results displayed in Figures 10 (a) and 10 (b) pertain to the probability density and the cumulative distribution, respectively. A comparison of the corresponding results displayed in Figures 8(a), 8(b), 10(a) and 10(b) reveals that the presence of side-group defects and the application of preludial axial compression result in a reduction in the mean value $(4.86 \mathrm{GPa})$ of the fiber longitudinal-tensile strength. In addition, the distribution standard deviation is increased from $\sim 0.40 \mathrm{GPa}$ (for the case of the same fibers which were not subjected to preludial axial compression) to $\sim 0.52 \mathrm{GPa}$ (in the present case). This finding suggests that, in the case of precompressed fibers, there is a higher probability for finding fibers with relatively low longitudinal-tensile strength.

The results presented in this section suggest that the nature (i.e., chain end versus side group) of the defects, as well as the defect concentration/cluster-size, may play a key role in the effect of preludial axial compression on the residual longitudinal-tensile strength of the PPTA fibers. That is, preludial axial compression has substantially smaller detrimental effect on the fiber longitudinal-tensile strength in the case of chain-end defects. In sharp contrast, this negative effect can become significant when PPTA fibers contain sidegroup defects. 


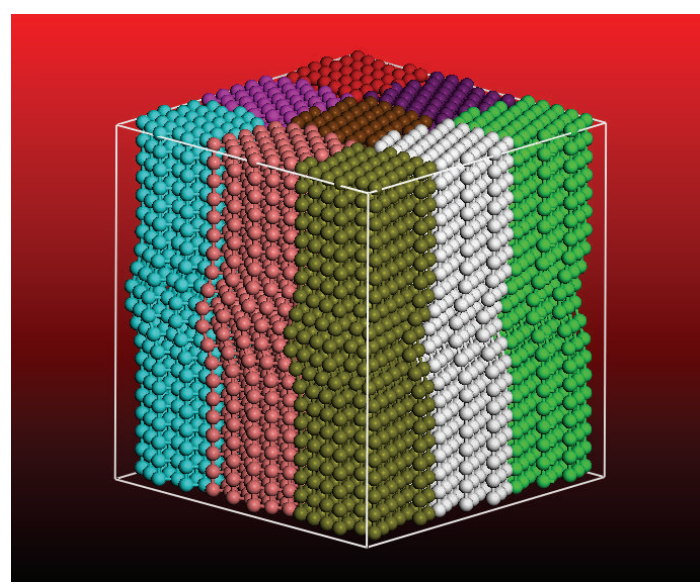

(a)

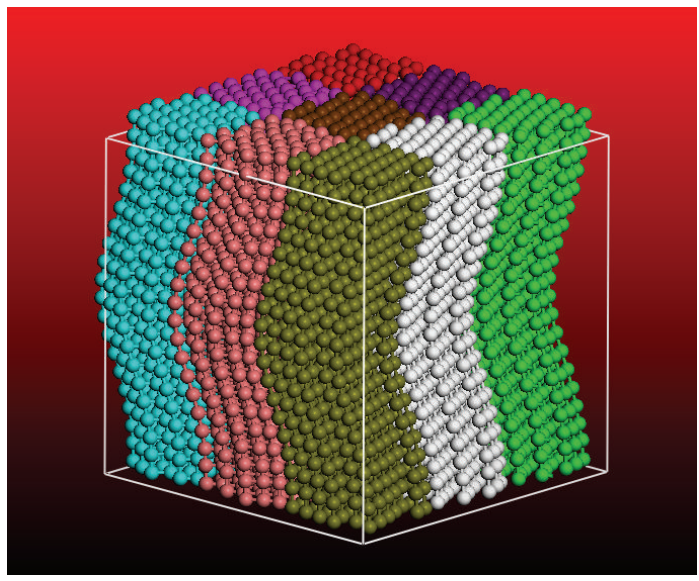

(c)

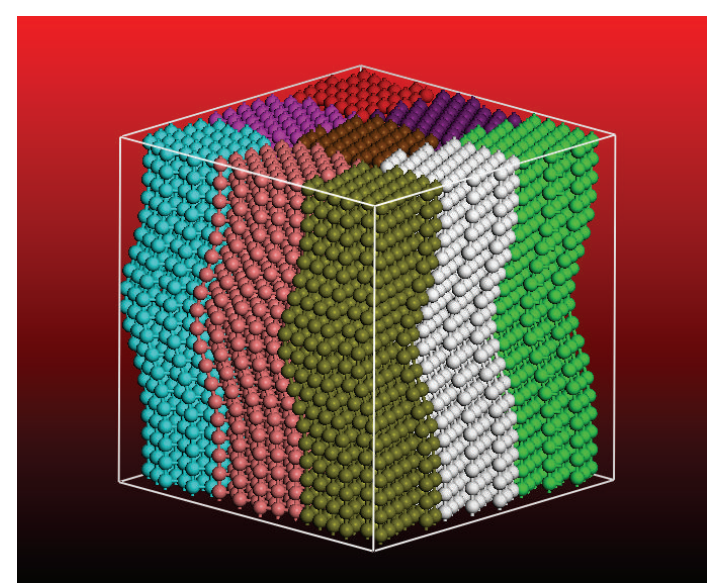

(b)

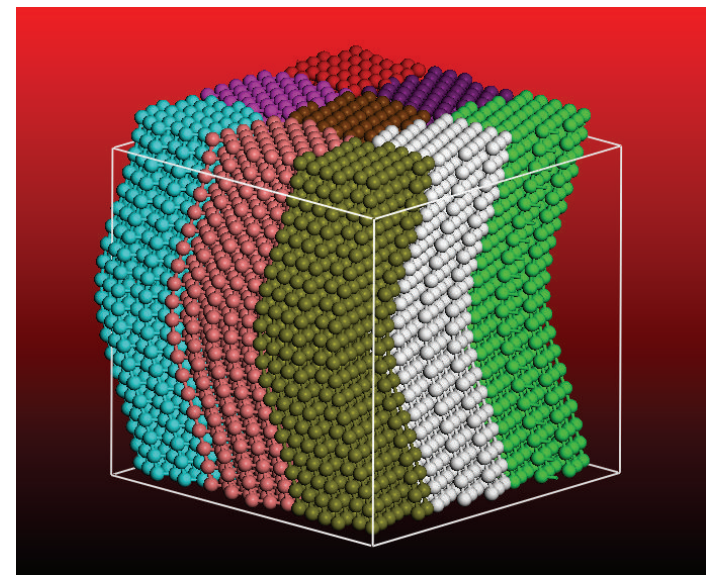

(d)

FIGURE 9: Coarse-grained microstructure, during axial compression, within the computational cell containing (initially) defective virgin PPTA fiber at the longitudinal stretch values of: (a) 0.975 ; (b) 0.950 ; (c) 0.925 ; and (d) 0.900 . The fiber within the computational cell contains a cluster of side-group defects.

\section{Summary and Conclusions}

Based on the results obtained in the present work, the following summary remarks and main conclusions can be drawn.

(1) The effect of prior axial compression on the residual longitudinal-tensile strength of defect-free and defective $p$-phenylene terephthalamide (PPTA) fibers is investigated computationally using coarse-grained molecular statics and dynamics simulation methods. To construct the coarse-grained PPTA-fiber model, the results of a companion all-atom molecular-level computational analysis are utilized.

(2) In the case of defect-free PPTA fibers, the longitudinal-tensile strength was found to be essentially a deterministic quantity. In sharp contrast, in defective PPTA fibers, the same quantity was found to be of a stochastic character and to possess a characteristic probability density function.

(3) Preludial compression was found to alter the loworder moments of this distribution function, and the extent of these alterations was found to be dependent on the defect type and concentration. Specifically, chain-end defects are found to make this alteration small, while side groups are observed to increase the extent of these alterations. In the case of PPTA fibers containing side-group defects, preludial compression has been found to reduce the mean value of the fiber longitudinal-tensile strength and to increase the variance/standard-deviation of the associated probability density function. The extent of these changes was observed to increase with the extent of preludial axial compression.

(4) The results obtained clearly reveal that the quality control related to the precursor materials, synthesis conditions, and fiber-spinning process must be given particular attention, since the accompanying inadequacies may result in PPTA fibers with inferior mechanical properties.

(5) The results also point out that various textilemanufacturing processes used in the fabrication of high-performance protective systems may degrade 


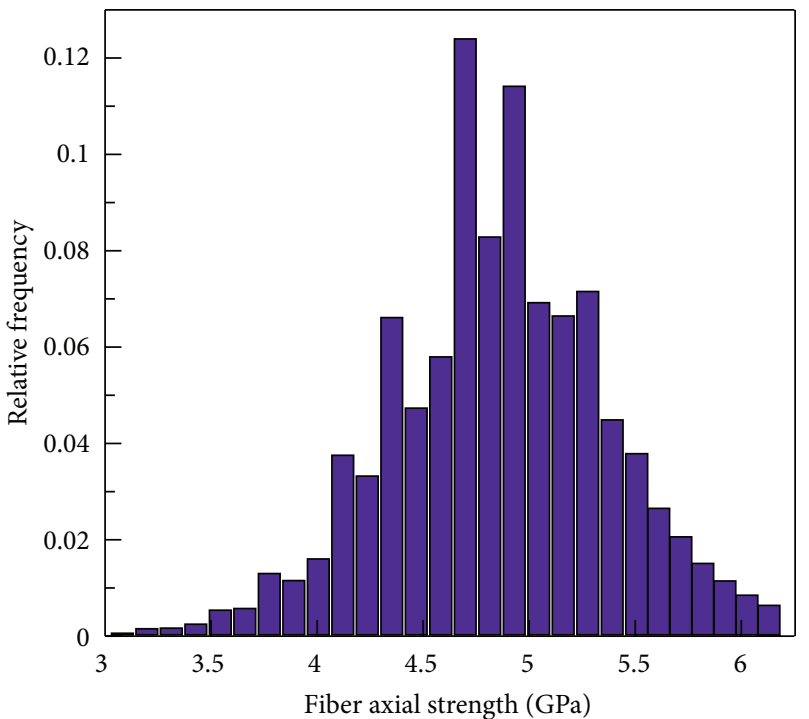

(a)

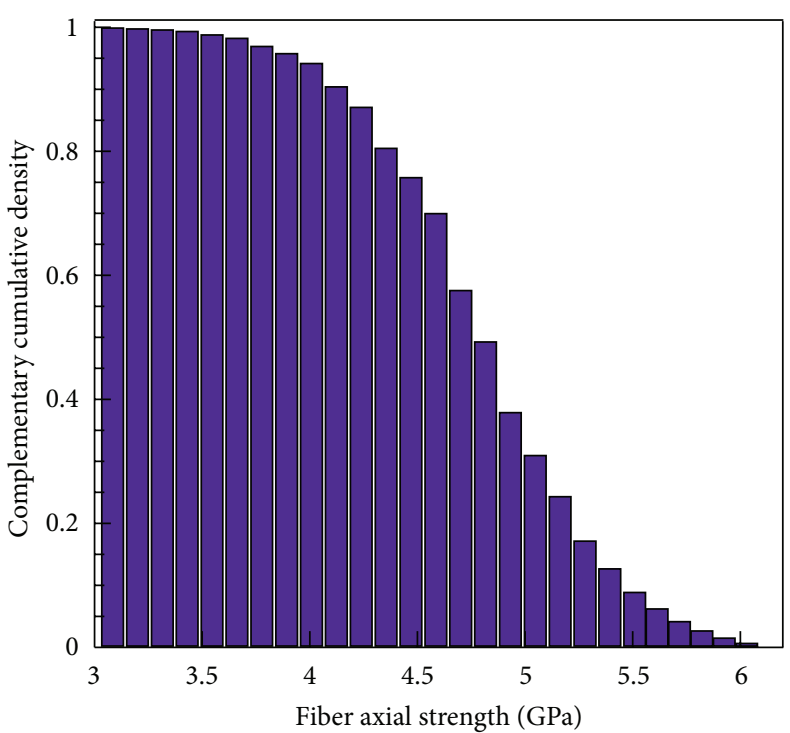

(b)

Figure 10: (a) Longitudinal-tensile-strength relative frequency (i.e., probability density function) and (b) the corresponding complementary cumulative density function for PPTA-fibers containing side-group defects after preludial axial compression to a maximum strain of -0.105.

the properties of PPTA fibers relative to their as-synthesized/as-spun levels.

\section{Acknowledgments}

The material presented in this paper is based on work supported by the Army Research Office (ARO) research contract entitled "Multi-length Scale Material Model Development for Armor-grade Composite," Contract no. W911NF-09-1-0513. The authors are indebted to Dr. Larry Russell of ARO for his continuing support and interest in the present work.

\section{References}

[1] M. Grujicic, G. Arakere, T. He et al., "A ballistic material model for cross-plied unidirectional ultra-high molecularweight polyethylene fiber-reinforced armor-grade composites," Materials Science and Engineering A, vol. 498, no. 1-2, pp. 231241, 2008.

[2] M. Grujicic, G. Arakere, T. He, W. C. Bell, P. S. Glomski, and B. A. Cheeseman, "Multi-scale ballistic material modeling of cross-plied compliant composites," Composites Part B, vol. 40, no. 6, pp. 468-482, 2009.

[3] M. Grujicic, P. S. Glomski, T. He, G. Arakere, W. C. Bell, and B. A. Cheeseman, "Material modeling and ballistic-resistance analysis of armor-grade composites reinforced with highperformance fibers," Journal of Materials Engineering and Performance, vol. 18, no. 9, pp. 1169-1182, 2009.

[4] M. Grujicic, W. C. Bell, G. Arakere, T. He, X. Xie, and B. A. Cheeseman, "Development of a meso-scale material model for ballistic fabric and its use in flexible-armor protection systems," Journal of Materials Engineering and Performance, vol. 19, no. 1, pp. 22-39, 2010.

[5] M. Grujicic, W. C. Bell, P. S. Glomski, B. Pandurangan, C.F. Yen, and B. A. Cheeseman, "Filament-level modeling of aramid-based high-performance structural materials," Journal of Materials Engineering and Performance, vol. 20, no. 8, pp. 1401-1413, 2011.

[6] M. Grujicic, P. S. Glomski, B. Pandurangan, W. C. Bell, C.-F. Yen, and B. A. Cheeseman, "Multi-length scale computational derivation of Kevlar® yarn-level material model," Journal of Materials Science, vol. 46, no. 14, pp. 4787-4802, 2011.

[7] M. Grujicic, B. Pandurangan, J. S. Snipes, C.-F. Yen, and B. A. Cheeseman, "Multi-length scale enriched continuumlevel material model for Kevlar-fiber reinforced polymer-matrix composites," Journal of Materials Engineering and Performance, vol. 22, pp. 681-695, 2013.

[8] M. Grujicic, S. Ramaswami, J. S. Snipes et al., "Molecular-level computational investigation of mechanical transverse behavior of p-phenylene terephthalamide (PPTA) fibers," Multidiscipline Modeling in Materials and Structures, 2013.

[9] R. Edmunds and M. A. Wadee, "On kink banding in individual PPTA fibres," Composites Science and Technology, vol. 65, no. 78, pp. 1284-1298, 2005.

[10] M. G. Dobb, D. J. Johnson, and B. P. Saville, "Compressional behaviour of Kevlar fibres," Polymer, vol. 22, no. 7, pp. 960-965, 1981.

[11] M. Grujicic, G. Arakere, H. Nallagatla, W. C. Bell, and I. Haque, "Computational investigation of blast survivability and off-road performance of an up-armoured high-mobility multi-purpose wheeled vehicle," Journal of Automobile Engineering, vol. 223, no. 3, pp. 301-325, 2009.

[12] M. Grujicic, B. Pandurangan, U. Zecevic, K. L. Koudela, and B. A. Cheeseman, "Ballistic performance of alumina/S-2 glassreinforced polymer-matrix composite hybrid lightweight armor against armor piercing (ap) and non-AP projectiles," Multidiscipline Modeling in Materials and Structures, vol. 3, no. 3, pp. 287-312, 2007.

[13] M. Grujicic, B. Pandurangan, K. L. Koudela, and B. A. Cheeseman, "A computational analysis of the ballistic performance of 
light-weight hybrid composite armors," Applied Surface Science, vol. 253, no. 2, pp. 730-745, 2006.

[14] M. Grujicic, R. Yavari, S. Ramaswami, J. S. Snipes, C. -F. Yen, and B. A. Cheeseman, "Molecular-level study of the effect of prior axial compression/torsion on the axial-tensile strength of PPTA fibers," Journal of Materials Engineering and Performance, 2013.

[15] T. Takahashi, M. Miura, and K. Sakurai, "Deformation band studies of axially compressed poly(p-phenylene terephthalamide) fiber," Journal of Applied Polymer Science, vol. 28, no. 2, pp. 579-586, 1983.

[16] S. J. Deteresa, S. R. Allen, R. J. Farris, and R. S. Porter, "Compressive and torsional behaviour of Kevlar 49 fibre," Journal of Materials Science, vol. 19, no. 1, pp. 57-72, 1984.

[17] 2013, http://accelrys.com/products/datasheets/materials-visualizer.pdf.

[18] M. Grujicic, B. Pandurangan, A. E. King, J. Runt, J. Tarter, and G. Dillon, "Multi-length scale modeling and analysis of microstructure evolution and mechanical properties in polyurea," Journal of Materials Science, vol. 46, no. 6, pp. 1767-1779, 2011.

[19] M. Grujicic, J. S. Snipes, S. Ramaswami et al., "Coarse-grained molecular-level analysis of polyurea properties and shockmitigation potential," Journal of Materials Engineering and Performance, vol. 22, pp. 1964-1981, 2013.

[20] M. Grujicic, T. He, B. Pandurangan, F. R. Svingala, G. S. Settles, and M. J. Hargather, "Experimental characterization and material-model development for microphase-segregated polyurea: an overview," Journal of Materials Engineering and Performance, vol. 21, no. 1, pp. 2-16, 2012.

[21] 2013, http://accelrys.com/products/datasheets/discover.pdf.

[22] J. A. Newell and M. T. Sagendorf, "Experimental verification of the end-effect Weibull model as a predictor of the tensile strength of Kevlar-29 (poly p-phenyleneterephthalamide) fibres at different gauge lengths," High Performance Polymers, vol. 11, no. 3, pp. 297-305, 1999.

[23] L. W. Steenbakkers and H. D. Wagner, "Elasticity and mechanical breakdown of Kevlar 149 aramid fibres by a probabilistic approach," Journal of Materials Science Letters, vol. 7, no. 11, pp. 1209-1212, 1988.

[24] W. F. Knoff, "A modified weakest-link model for describing strength variability of Kevlar aramid fibres," Journal of Materials Science, vol. 22, no. 3, pp. 1024-1030, 1987.

[25] M. Grujicic, T. He, B. Pandurangan, J. Runt, J. Tarter, and G. Dillon, "Development and parameterization of a timeinvariant (equilibrium) material model for segmented elastomeric polyureas," Journal of Materials: Design and Applications, vol. 225, no. 3, pp. 182-194, 2011.

[26] M. Grujicic, T. He, and B. Pandurangan, "Development and parameterization of an equilibrium material model for segmented polyurea," Multidiscipline Modeling in Materials and Structures, vol. 7, no. 2, pp. 96-114, 2011.

[27] M. Grujicic, G. Arakere, W. C. Bell et al., "Reliability-based design optimization for durability of ground vehicle suspension system components," Journal of Materials Engineering and Performance, vol. 19, no. 3, pp. 301-313, 2010.

[28] M. Grujicic, W. C. Bell, B. Pandurangan, and T. He, "Blastwave impact-mitigation capability of polyurea when used as helmet suspension pad material," Materials and Design, vol. 31, pp. 4050-4065, 2010.

[29] M. Grujicic, B. P. d'Entremont, B. Pandurangan et al., "A study of the blast-induced brain white-matter damage and the associated diffuse axonal injury," Multidiscipline Modeling in Materials and Structures, vol. 8, no. 2, pp. 213-245, 2012. 

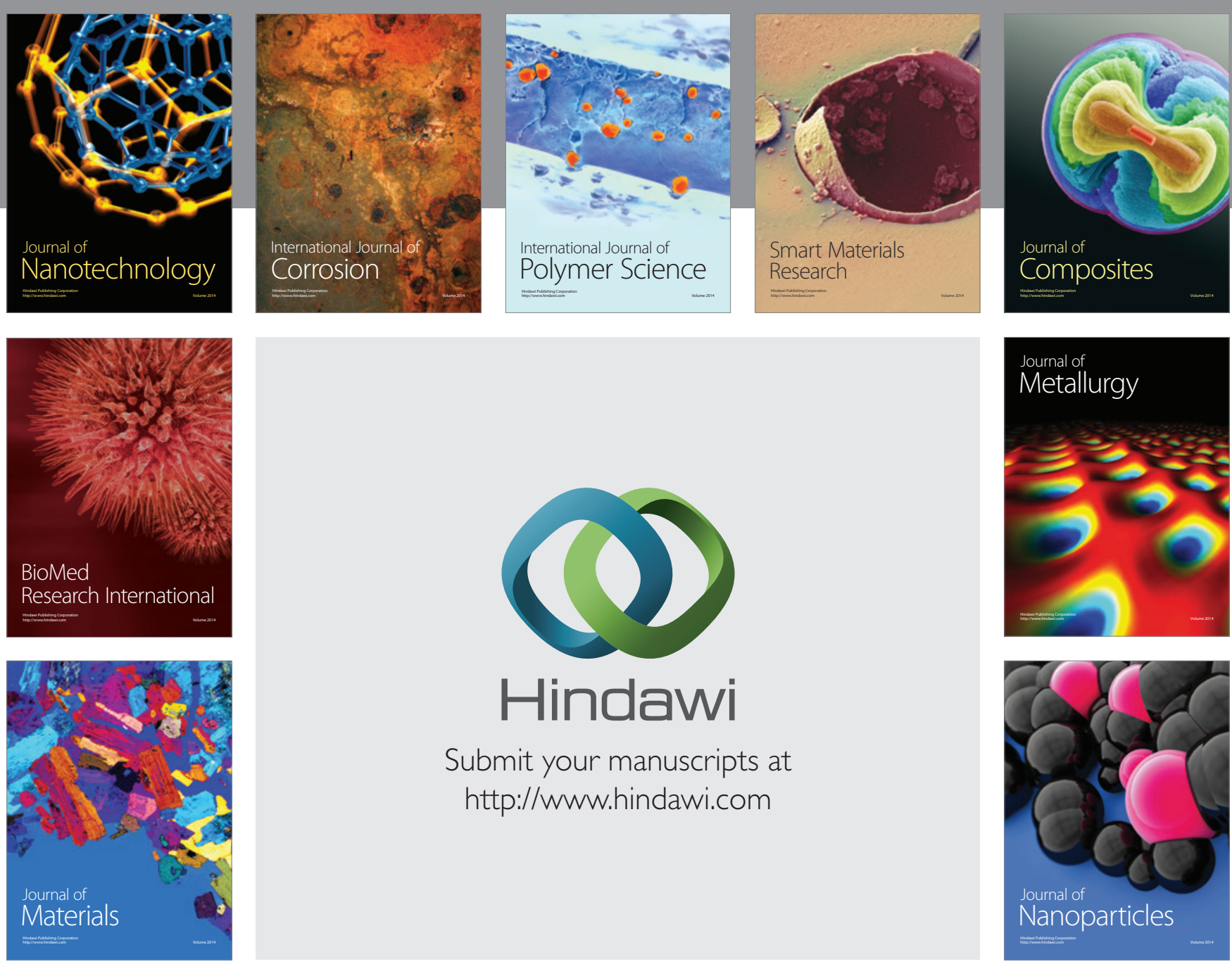

Submit your manuscripts at http://www.hindawi.com
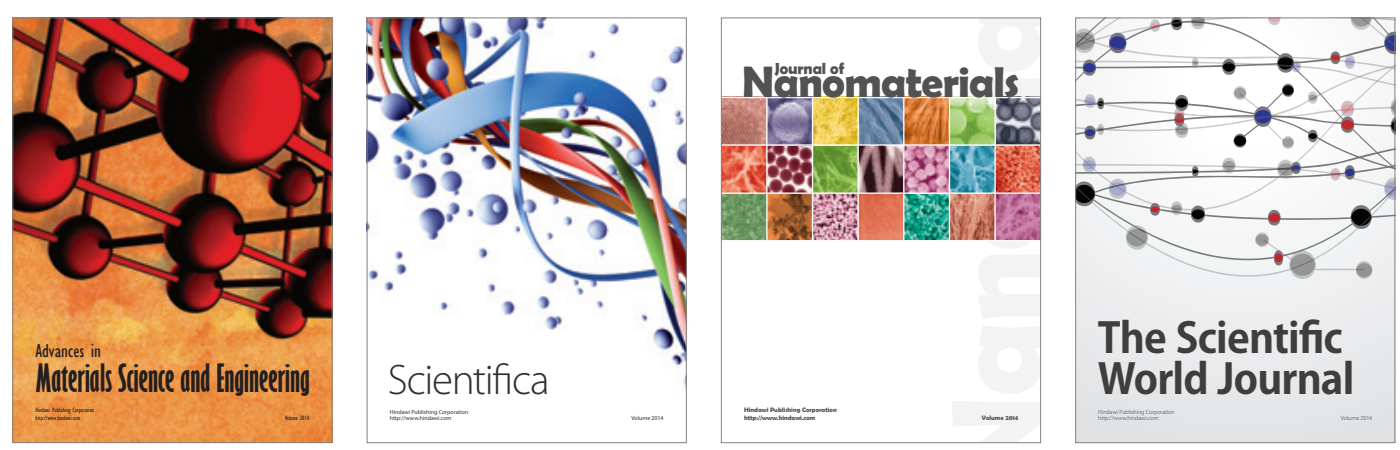

\section{The Scientific World Journal}
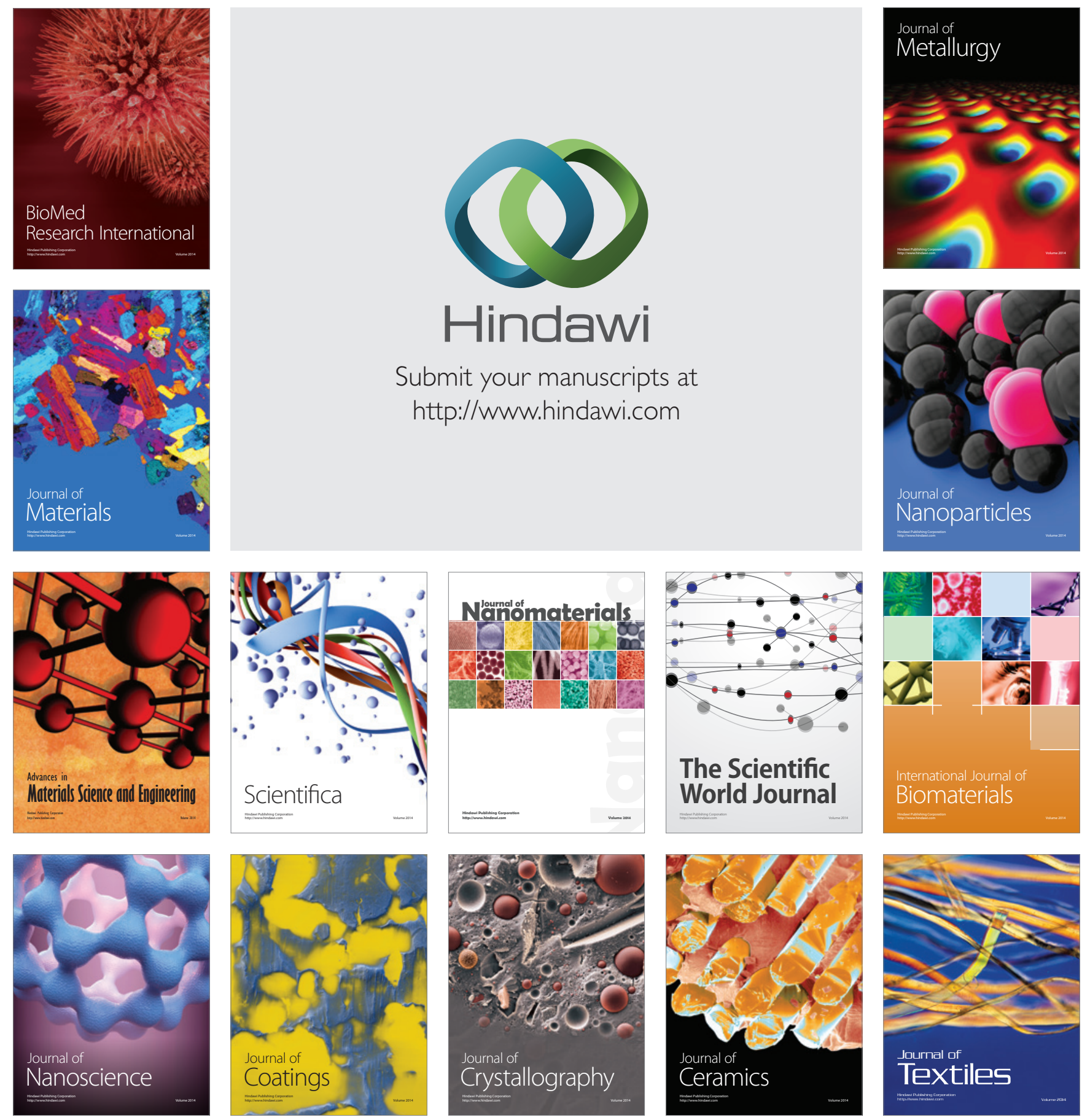